\title{
Association between overweight/obesity and eating habits while watching television among primary-school children in the city of Shiraz, Iran
}

\author{
Saeed Ghobadi ${ }^{1}$, Julia O Totosy de Zepetnek ${ }^{2}$, Zeinab Hemmatdar ${ }^{3}$, Nick Bellissimo ${ }^{4}$, \\ Reza Barati ${ }^{3}$, Hoda Ahmadnia ${ }^{3}$, Mohammad Salehi-Marzijarani ${ }^{5}$ and Shiva Faghih ${ }^{1,6, *}$ \\ ${ }^{1}$ Nutrition Research Center, School of Nutrition and Food Sciences, Shiraz University of Medical Sciences, Razi Blvd, \\ Shiraz, Islamic Republic of Iran: ${ }^{2}$ Faculty of Kinesiology \& Health Studies, University of Regina, Regina, SK, Canada: \\ ${ }^{3}$ Student Research Committee, School of Nutrition and Food Sciences, Shiraz University of Medical Sciences, Shiraz, \\ Islamic Republic of Iran: ${ }^{4}$ Faculty of Community Services, School of Nutrition, Ryerson University, Toronto, ON, \\ Canada: ${ }^{5}$ Department of Biostatistics, Faculty of Medicine, Shiraz University of Medical Sciences, Shiraz, Islamic \\ Republic of Iran: 'Department of Community Nutrition, School of Nutrition and Food Sciences, Shiraz University of \\ Medical Sciences, Shiraz, Islamic Republic of Iran
}

Submitted 18 April 2017: Final revision received 21 September 2017: Accepted 6 October 2017: First published online 27 November 2017

\begin{abstract}
Objective: It has been reported that television (TV) viewing is associated with childhood obesity in Western countries. The present study aimed to investigate the relationship between obesity and eating habits while watching TV among primaryschool children in the Middle East.

Design: Cross-sectional.

Setting: Children were recruited from primary schools of four educational districts in Shiraz, Iran. Anthropometric indices of mass (kg) and height (m) were measured, and BMI (percentile) was calculated. Demographic characteristics, TV viewing behaviours and physical activity data were collected from parents during face-to-face interviews and a $3 \mathrm{~d}$ dietary record was completed.

Subject: Children ( $n$ 607) aged 6-10 years.

Results: Mean (SD) age of children was 8.16 (1.37) years, of whom 9.1 and $8.4 \%$ were overweight and obese, respectively. Children who spent $\geq 2 \mathrm{~h}$ watching TV on weekdays (OR $=1.99 ; 95 \%$ CI 1.09, 3.60) and weekend days (OR $=1.86 ; 95 \%$ CI 1.01, 3.43) had higher odds of being obese, even after adjusting for physical activity. Children who ate breakfast while watching TV had higher odds of being overweight $v$. those who did not watch TV while eating breakfast (OR $=2 \cdot 70 ; 95 \%$ CI 1.02, 7.60). There were no associations between TV viewing during other meals (lunch and dinner) and overweight/obesity.

Conclusions: TV viewing for $\geq 2 \mathrm{~h}$ daily increases the risk of being obese in Iranian children aged 6-10 years, independent of physical activity. Further, breakfast consumption while watching TV may increase the risk of overweight/obesity, independent of total TV viewing time.
\end{abstract}

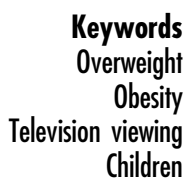

Overweight/obesity in children has become a major challenge for public health ${ }^{(1)}$. As one of the leading risk factors for mortality, obesity is responsible for $5 \%$ of deaths worldwide ${ }^{(2)}$. Childhood obesity can increase the risk of CVD and diabetes in later life ${ }^{(3)}$, reduces quality of life and life expectancy, and costs the health-care system billions of dollars per year owing to its secondary complications $^{(4)}$. According to studies conducted in Iran among children and adolescents aged 6-18 years in 2011-2012, the prevalence of overall and abdominal obesity is 11.89 and $19 \cdot 21 \%$, respectively ${ }^{(5)}$.
Sedentary behaviour (defined as energy expenditure of less than 1.5 MET (metabolic equivalents of task)) has recently been identified as a health risk factor ${ }^{(6)}$. Television (TV) viewing is one of the most prevalent sedentary activities in both children and adults ${ }^{(7)}$, and compared with other sedentary activities such as reading and writing, TV viewing is associated with a lower metabolic rate ${ }^{(8)}$. A meta-analysis performed on prospective cohort studies reported that prolonged TV viewing was associated with increased risk of CVD, type 2 diabetes and other causes of death ${ }^{(9)}$. TV viewing is thought to contribute to 
overweight/obesity by either displacing physical activity and decreasing metabolic rate, or increasing energy intake, or both. Increases in consumption of junk foods and soft drinks in parallel with a reduction of fruit and vegetable consumption as a result of watching TV has been reported $^{(10,11)}$. Observational studies have reported a positive relationship between TV viewing during meal consumption and poor diet, as well as between TV viewing and increased $\mathrm{BMI}^{(7,11,12)}$. Studies assessing the relationship between eating meals while watching TV and obesity in children have yielded contradictory findings. For instance, some studies have reported that obesity is related to eating while watching TV in Canadian and European cohorts ${ }^{(4,10)}$, while other studies reported no associations in American and European cohorts ${ }^{(13,14)}$.

Iran is currently experiencing an epidemiological transition in conjunction with a negative nutritional transition that is contributing to increasing prevalence of nutritional disorders among children ${ }^{(15)}$. In addition, TV viewing time is increasing among Iranian children; however, no studies to date have investigated the consequences of increased TV viewing time on food habits, nutritional status and risk of being overweight/obese among Iranian children and/or adolescents. The purpose of the present study was to investigate the relationship between eating habits while watching TV and overweight/obesity in children in Shiraz, one of the large cities of Iran.

\section{Methods}

\section{Participants}

The present study was designed to evaluate the association between overweight/obesity and eating habits while watching TV among primary-school children/adolescents in urban areas of Shiraz in the south of Iran. Data collection took place in Shiraz, Iran, from October 2015 to June 2016. Children were randomly selected using stratified multistage sampling methods from a list of all schools (private and public) in Shiraz provided by the Department of Education. Four educational districts of Shiraz were selected as strata and then four primary schools were randomly selected from each stratum. Two classes were randomly selected from each school and all students in selected classrooms were invited to participate in the study. Children were eligible if they were between 6 and 10 years old and studied in primary schools in Shiraz, Iran. Children with incomplete information, serious health problems, or any type of physical or mental impairments were excluded from the final analyses.

The study protocol was described to managers of selected schools and then invitations were sent to parents for an interview at the school. All parents were free to refuse participation. Anthropometric assessments and interviews were conducted at the school over two study visits. During the first visit, all procedures were explained, informed assent and consent were obtained from the children and parents, respectively, and all questionnaires were completed via face-to-face interviews with parents. On the second visit, anthropometric indices were measured and parents were given a $3 \mathrm{~d}$ food record to complete within the following week. Shiraz University of Medical Sciences Research Ethics Committee approved the study procedures (no. 94-01-84-9888).

\section{Antbropometric measurements}

All anthropometric measurements were obtained via standardized procedures by trained researchers. Children's mass $(\mathrm{kg})$ was measured in light clothing with a digital body composition monitor (BF511; OMRON, China) to the nearest $0 \cdot 1 \mathrm{~kg}$. Height $(\mathrm{cm})$ was measured barefoot to the nearest $0.5 \mathrm{~cm}$ using a non-elastic tape measure. Anthropometric measurements were obtained twice, and the mean values were recorded. BMI percentiles were calculated by the WHO AnthroPlus software version 1.0.4. According to the WHO definitions, underweight was defined as a BMI less than the 5 th percentile, overweight as a BMI at or above the 85th percentile and below the 95th percentile, and obesity as a BMI at or above the 95th percentile for children of the same age and $\operatorname{sex}^{(16)}$.

\section{Assessment of physical activity}

The Quantification de L'Activite Physique en Altitude chez les Enfants questionnaire (QAPACE), appropriate for estimating physical activity among 6-18-year-old children, was completed via a face-to-face interview with parents. The questionnaire was easy to use and convenient, measuring activities at school, on vacation and at home. Reliability and validity of this questionnaire have been assessed in Iranian children and adolescents ${ }^{(17)}$.

\section{Assessment of socio-economic status}

Socio-economic status of the families was estimated by the validated Family Affluence Scale (FAS) that included four questions: (i) 'Does your family have a car?'; (ii) 'Does the child have a separate bedroom?'; (iii) 'How many times a year does your family go on trips?'; and (iv) 'How many computers/laptops does your family have?' Children were classified as low, medium or high economic status ${ }^{(18)}$.

\section{Dietary intake}

Energy intake was measured using a $3 \mathrm{~d}$ dietary record (two weekdays and one weekend day), completed by parents. Parents were also asked to specify which of the meals and snacks were eaten while watching TV. Using household measuring cups/spoons and food models/ photographs, experienced nutritionists educated parents on how to complete the $24 \mathrm{~h}$ dietary records. Mixed foods were converted into their ingredients according to each parent's report on the amount of the food item consumed. Furthermore, parents were asked to write down the food preparation method (i.e. frying, boiling or cooking). All dietary intakes were analysed using Nutritionist 
4 software (First Databank Inc., Hearst Corp., San Bruno, CA, USA).

\section{Assessment of television viewing behaviours}

Frequency of meals consumed while watching TV was determined with the question, 'How often do your children eat their meals while TV viewing? (Meals include breakfast, lunch and dinner)', using the following response options: 'always', 'often', 'sometimes' and 'never'. TV viewing time during weekends and weekdays was assessed by separate questions, 'How many hours does your child spend TV viewing during weekends/weekdays?', with nine response options: '30 minutes/day', '1 hour/day', '1.5 hours/day', '2 hours/day', ..., '4 hours/day ${ }^{(10)}$.

Parents were also asked how often their children consumed five different types of snacks in front of the TV ('potato chips', 'fried chicken', 'fruit, biscuits, chocolate and candy', 'ice cream' and 'fast food') and two types of drinks ('soft drinks' and 'lemonade'). The response options included: 'never', 'less than once a week', '1 or 2 times a week', ' 3 or 4 times a week' and 'almost every day'(19).

\section{Statistical analyses}

Percentage of energy provided by different macronutrients in each meal for two conditions (TV on and TV off) were compared using $t$ tests. Multinomial regression analyses were used to calculate the odds of being overweight/ obese taking account of the frequency of eating junk food and meals (breakfast, lunch and dinner) while watching TV on both weekdays and weekend days. Model 1 adjusted for sex, birth order and income; model 2 adjusted for the same factors and further included physical activity; model 3 further included energy intake; and model 4 further included TV viewing time. Other possible confounding variables (e.g. parents' education, family size and child custody) were not entered in the multivariate analyses because their association with BMI percentile had a $P$ value of $>0 \cdot 1$. A weighted kappa $\left(\kappa_{\mathrm{w}}\right)$ agreement statistic was used to confirm the level of agreement between the different measurement methods of determining frequency of eating meals while watching TV (dietary records $v$. survey questionnaire). Statistical analyses were performed using the statistical software package IBM SPSS Statistics version 19, with a level of significance of $P<0.05$.

\section{Results}

Of the 647 students invited to participate in the study, fourteen refused to participate, eighteen had incomplete data for TV viewing time or dietary records, and nine were excluded due to chronic diseases that may have affected study results such as diabetes and thyroid disorders. After excluding missing values and incomplete data, a sample of 607 primary-school children ( 276 boys and 331 girls) between the ages of 6 and 10 years were included for analyses.
The response rate of study was $93.8 \%$. There were no significant differences in sociodemographic characteristics between the participants who were included or excluded from the final analyses. Kappa agreement tests indicated good agreement between questions pertaining to snacks/ meals eaten while watching TV from the dietary records and survey questions $\left(\kappa_{\mathrm{w}, \text { breakfast }}=0.69, \kappa_{\mathrm{w}, \text { lunch }}=0.63, \kappa_{\mathrm{w}, \text { dinner }}=\right.$ $0.74, P<0.001)$. Demographic and socio-economic characteristics of the children $(n 607)$ are shown in Table 1. Mean age was 8.16 (SD 1.37) years, with more than half being female (54.5\%) and $17.5 \%$ being overweight/obese. Most of the parents had high school education, and almost half of the children were from families with medium economic status.

On weekdays and weekend days, 31.6 and $35.9 \%$ of the children spent $\geq 2 \mathrm{~h}$ watching TV, respectively (Table 2 ). We found that $20 \cdot 7,19 \cdot 3$ and $21 \cdot 1 \%$ of the children always ate their breakfast, lunch and dinner while watching TV, respectively. No differences were found between girls and boys in terms of TV viewing behaviours, therefore data from both sexes were pooled to increase power.

Table 3 reports the odds of being overweight/obese in relation to TV viewing. Children who spent $\geq 2 \mathrm{~h}$ watching TV on weekdays had higher odds of being obese compared with children who spent $<2 \mathrm{~h}$ watching TV on weekdays ( $\mathrm{OR}=2 \cdot 06 ; 95 \% \mathrm{CI} 1 \cdot 15,3 \cdot 70)$. This association remained significant after adjustment for factors in model 1 of sex, birth order and income (OR $=1.97 ; 95 \%$ CI 1.09, 3.60), and in model 2 further including physical activity $(\mathrm{OR}=1 \cdot 86 ; 95 \% \mathrm{CI} 1 \cdot 01,3.43)$. When further including energy intake in model 3 , the association was no longer significant. However, on weekend days, the same association remained significant when including energy intake in model $3(\mathrm{OR}=2 \cdot 44 ; 95 \% \mathrm{CI} 1 \cdot 03,5 \cdot 70)$. Children who always ate breakfast while watching TV had a 2.70 higher odds of being overweight compared with children who never ate breakfast in front of the TV (95\% CI 1.02, 7.60), even after adjusting for all confounding variables in models $1-4$. There was no significant relationship between consuming junk food or other meals while watching TV and the odds of being overweight/obese.

Table 4 shows associations between different macronutrient intakes and TV viewing. Children who ate breakfast while watching TV consumed a higher percentage of energy intake from carbohydrates $(P=0.02)$ and a lower percentage of energy intake from protein $(P=0 \cdot 03)$. Children who had snacks while watching TV consumed a higher percentage of energy intake from fats compared with children who had snacks without watching TV. No differences were observed between other meals (lunch or dinner) and percentage of energy intake from macronutrients with or without TV.

\section{Discussion}

The present study is the first among children from the Middle East investigating the relationship between mealtime TV viewing and overweight/obesity. Results from our 
Table 1 Demographic and socio-economic characteristics of participants by sex: primary-school children ( $n$ 607) aged 6-10 years, Shiraz, Iran, October 2015-June 2016

\begin{tabular}{|c|c|c|c|c|c|c|}
\hline \multirow[b]{2}{*}{ Variable } & \multicolumn{2}{|c|}{ Boys ( $n$ 276) } & \multicolumn{2}{|c|}{ Girls (n 331) } & \multicolumn{2}{|c|}{ Total ( $n$ 607) } \\
\hline & Mean or $n$ & SD or $\%$ & Mean or $n$ & SD or $\%$ & Mean or $n$ & SD or $\%$ \\
\hline Age (years), mean and SD & 8.03 & 1.43 & 8.26 & 1.30 & $8 \cdot 16$ & 1.37 \\
\hline BMl $\left(\mathrm{kg} / \mathrm{m}^{2}\right)$, mean and SD & $16 \cdot 45$ & 2.94 & $16 \cdot 00$ & $2 \cdot 70$ & $16 \cdot 20$ & 2.80 \\
\hline Underweight, $n$ and $\%$ & 37 & 13.4 & 39 & 11.8 & 76 & $12 \cdot 5$ \\
\hline Normal weight, $n$ and $\%$ & 178 & 64.5 & 247 & 74.6 & 425 & $70 \cdot 0$ \\
\hline Overweight, $n$ and $\%$ & 30 & 10.9 & 25 & 7.6 & 55 & $9 \cdot 1$ \\
\hline Obese, $n$ and $\%$ & 31 & $11 \cdot 2$ & 20 & $6 \cdot 0$ & 51 & 8.4 \\
\hline \multicolumn{7}{|l|}{ Birth order, $n$ and $\%$} \\
\hline 1 & 172 & $62 \cdot 3$ & 173 & $52 \cdot 3$ & 345 & $56 \cdot 8$ \\
\hline 2 & 73 & $26 \cdot 4$ & 111 & 33.5 & 184 & $30 \cdot 3$ \\
\hline 3 & 23 & 8.3 & 32 & 9.7 & 55 & $9 \cdot 1$ \\
\hline$>3$ & 8 & $2 \cdot 9$ & 15 & 4.5 & 23 & 3.8 \\
\hline \multicolumn{7}{|l|}{ Family size, $n$ and $\%$} \\
\hline$\leq 4$ & 211 & $76 \cdot 4$ & 249 & $75 \cdot 2$ & 460 & $75 \cdot 8$ \\
\hline$\geq 5$ & 65 & 23.6 & 82 & 24.8 & 147 & $24 \cdot 2$ \\
\hline \multicolumn{7}{|l|}{ Child lives with, $n$ and \% } \\
\hline Both parents & 259 & 93.8 & 312 & 94.3 & 571 & $94 \cdot 1$ \\
\hline Father & 5 & 1.8 & 6 & 1.8 & 11 & 1.8 \\
\hline Mother & 11 & 4.0 & 11 & 3.3 & 22 & 3.6 \\
\hline Other & 1 & 0.4 & 2 & 0.6 & 3 & 0.5 \\
\hline \multicolumn{7}{|l|}{ Mother's education, $n$ and $\%$} \\
\hline Elementary & 94 & 34.6 & 120 & $36 \cdot 6$ & 214 & $35 \cdot 7$ \\
\hline Secondary & 143 & 52.6 & 163 & $49 \cdot 7$ & 306 & 51.0 \\
\hline Academic & 35 & $12 \cdot 9$ & 45 & $13 \cdot 7$ & 80 & $13 \cdot 3$ \\
\hline \multicolumn{7}{|l|}{ Father's education, $n$ and $\%$} \\
\hline Elementary & 89 & 33.7 & 108 & 33.4 & 197 & 33.6 \\
\hline Secondary & 129 & 48.9 & 139 & 43.0 & 268 & $45 \cdot 7$ \\
\hline Academic & 46 & 17.4 & 76 & 23.5 & 122 & $20 \cdot 8$ \\
\hline \multicolumn{7}{|l|}{ Socio-economic status, $n$ and $\%$} \\
\hline Low & 114 & $41 \cdot 3$ & 129 & $39 \cdot 0$ & 243 & $40 \cdot 0$ \\
\hline Medium & 121 & 43.8 & 149 & $45 \cdot 0$ & 270 & 44.5 \\
\hline High & 41 & 14.9 & 53 & $16 \cdot 0$ & 94 & $15 \cdot 5$ \\
\hline Physical activity index (MET-h/week), mean and SD & $31 \cdot 8$ & $5 \cdot 36$ & $24 \cdot 0$ & $10 \cdot 01$ & $27 \cdot 5$ & 8.22 \\
\hline
\end{tabular}

MET, metabolic equivalents of task.

Elementary education = less than 5 years of education; secondary education $=12$ years of education; academic $=$ having a university degree.

$P$ values for differences between boys and girls were not significant.

study indicate that both total TV viewing time on weekdays and weekend days, as well as eating habits while watching TV, are associated with overweight/obesity among Iranian children aged 6-10 years. Our major and novel findings are: (i) prolonged TV viewing on weekdays or weekend days increases the odds of obesity, independent of physical activity; and (ii) consuming breakfast in front of the TV increases the odds of overweight among Iranian children aged 6-10 years, independent of total TV viewing time.

Some clinical trial studies have reported no effect of TV viewing on overweight/obesity ${ }^{(20,21)}$. For example, a study conducted by Yilmaz et al. showed that reducing screen time in children aged 2-6 years for 9 months was not effective to decrease $\mathrm{BMI}^{(20)}$. However, several other studies have reported that TV viewing during childhood is associated with more weight gain and overweight in adulthood $^{(22,23)}$, and a recent meta-analysis showed that time spent watching TV was associated with increased risk for childhood obesity ${ }^{(24)}$. Results from the present study showed that increasing hours of time spent watching TV during weekdays and weekend days was associated with increased odds of overweight/obesity in Iranian children; these findings are in agreement with other cross-sectional studies examining the relationship between hours of TV viewing and overweight/obesity among children ${ }^{(25,26)}$. It is hypothesized that the relationship between TV viewing and overweight/obesity could be due to the amount of time spent watching TV displacing physical activity, increasing energy intake, or both. In the present study the association observed between TV viewing and overweight/obesity was independent of physical activity, suggesting that TV viewing time and physical activity are two distinct behaviours that may manipulate body mass via different metabolic mechanisms. Indeed, previous studies have reported independent associations between sedentary time and morbidity and mortality after adjusting for physical activity levels ${ }^{(27)}$. A study by Hamilton et al. suggested that prolonged sitting time could decrease lipoprotein lipase activity and may turn off the expression of some genes responsible for the maintenance of insulin sensitivity ${ }^{(28)}$. Another study reported that prolonged sedentary time might promote increased fat accumulation in the visceral, liver and heart regions ${ }^{(29)}$. 
Table 2 Television (TV) viewing behaviours of participants by sex: primary-school children $(n 607)$ aged $6-10$ years, Shiraz, Iran, October 2015-June 2016

\begin{tabular}{|c|c|c|c|c|c|c|}
\hline \multirow[b]{2}{*}{ TV exposure } & \multicolumn{2}{|c|}{ Boys ( $n$ 276) } & \multicolumn{2}{|c|}{ Girls (n 331) } & \multicolumn{2}{|c|}{ Total $(n 607)$} \\
\hline & $n$ & $\%$ & $n$ & $\%$ & $n$ & $\%$ \\
\hline \multicolumn{7}{|c|}{ Time spent watching TV on weekdays } \\
\hline$<2 \mathrm{~h}$ & 178 & 64.5 & 237 & 71.6 & 415 & 68.4 \\
\hline$\geq 2 \mathrm{~h}$ & 98 & 35.5 & 94 & 28.4 & 192 & 31.6 \\
\hline \multicolumn{7}{|c|}{ Time spent watching TV on weekend days } \\
\hline$<2 \mathrm{~h}$ & 175 & 63.9 & 210 & 64.4 & 385 & $64 \cdot 1$ \\
\hline$\geq 2 \mathrm{~h}$ & 100 & $36 \cdot 4$ & 116 & $35 \cdot 6$ & 217 & $35 \cdot 9$ \\
\hline \multicolumn{7}{|l|}{ Eats breakfast while watching TV } \\
\hline Always & 58 & 21.9 & 61 & $19 \cdot 6$ & 119 & $20 \cdot 7$ \\
\hline Often & 55 & $20 \cdot 8$ & 69 & $22 \cdot 2$ & 124 & $21 \cdot 5$ \\
\hline Sometimes & 91 & $34 \cdot 3$ & 136 & $43 \cdot 7$ & 227 & 39.4 \\
\hline Never & 61 & 23.0 & 45 & 14.5 & 106 & $18 \cdot 4$ \\
\hline \multicolumn{7}{|l|}{ Eats lunch while watching TV } \\
\hline Always & 68 & $24 \cdot 6$ & 49 & $14 \cdot 8$ & 117 & $19 \cdot 3$ \\
\hline Often & 85 & $30 \cdot 8$ & 85 & $25 \cdot 8$ & 170 & $28 \cdot 1$ \\
\hline Sometimes & 114 & 41.3 & 160 & 48.5 & 274 & $45 \cdot 2$ \\
\hline Never & 9 & 3.3 & 36 & $10 \cdot 9$ & 45 & 7.4 \\
\hline \multicolumn{7}{|l|}{ Eats dinner while watching TV } \\
\hline Always & 77 & $27 \cdot 9$ & 51 & $15 \cdot 5$ & 128 & $21 \cdot 1$ \\
\hline Often & 77 & 27.9 & 81 & 24.5 & 158 & $26 \cdot 1$ \\
\hline Sometimes & 109 & 39.5 & 164 & $49 \cdot 7$ & 273 & $45 \cdot 0$ \\
\hline Never & 13 & $2 \cdot 1$ & 34 & $5 \cdot 6$ & 47 & 7.8 \\
\hline \multicolumn{7}{|c|}{ Eats high-energy foods while watching TV } \\
\hline Almost every day & 38 & 13.8 & 29 & 8.8 & 67 & 11.0 \\
\hline Three or four times per week & 36 & 13.0 & 41 & $12 \cdot 4$ & 77 & $12 \cdot 7$ \\
\hline Once or twice per week & 91 & 33.0 & 123 & $37 \cdot 2$ & 214 & $35 \cdot 3$ \\
\hline Less than once per week & 77 & $27 \cdot 9$ & 87 & $26 \cdot 3$ & 164 & $27 \cdot 0$ \\
\hline Never & 34 & $12 \cdot 3$ & 51 & $15 \cdot 4$ & 85 & 14.0 \\
\hline
\end{tabular}

Eating meals while watching TV has been associated with overweight/obesity. While one early study in 2004 reported no association between food consumed during TV viewing and BMI in children ${ }^{(12)}$, several other studies have reported increased risk of overweight/obesity among children who ate their meals in front of the TV across eight European countries ${ }^{(30)}$. That large-scale cross-sectional study adjusted for possible confounding variables including age, country of residence, parents' education, dietary intakes and physical activity ${ }^{(30)}$. In another study by Dubois et $a l .{ }^{(31)}$, children who ate once daily or more while watching TV had higher mean BMI in comparison to children who reported less TV viewing during mealtimes; these findings were not adjusted for confounding variables. Results of our study investigating the relationship between eating meals while watching TV and weight status of children are somewhat in agreement with these studies. That is, we observed increased odds of overweight among children who consumed breakfast while watching TV, independent of the total TV viewing time. However, no association between other mealtime TV viewing (i.e. lunch, dinner) and overweight/obesity was observed. A study conducted by Wansink and van Kleef in American children reported similar findings of no association between eating dinner while watching TV and $\mathrm{BMI}^{(13)}$. On the other hand, a study examining meal consumption while watching TV in nine northern European countries showed no relationship between breakfast consumption during TV viewing and overweight/obesity, and a weak association between dinner consumption during TV viewing and overweight/obesity in children ${ }^{(14)}$.

The relationship between eating while watching TV and overweight/obesity may be attributed to diet quality; observational studies have reported a positive association between eating meals while watching TV and poor $\operatorname{diet}^{(30,32)}$. The majority of previous studies investigating diet quality and eating habits while watching TV in children did not investigate each meal separately ${ }^{(12,33,34)}$. A possible rationale for the discrepancy in findings between our study and previous research in European countries regarding breakfast consumption while watching TV could be due to different mealtimes and/or different types of TV programming. In the present study we found that children who ate breakfast while watching TV consumed a higher percentage of energy from carbohydrates and a lower percentage of energy from protein; however, we did not observe any relationship between total energy intake and TV viewing. In contrast to other countries where children consume about one-third of their daily energy intake at school from packed lunches and cafeteria-bought food ${ }^{(35)}$, primary-school children in Iran usually have their breakfast and lunch at home before going to school from 13.00 to 17.00 hours. Therefore, Iranian children have more time to eat breakfast while watching TV and are less supervised at breakfast time 


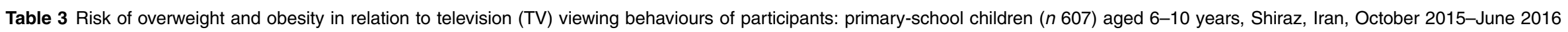

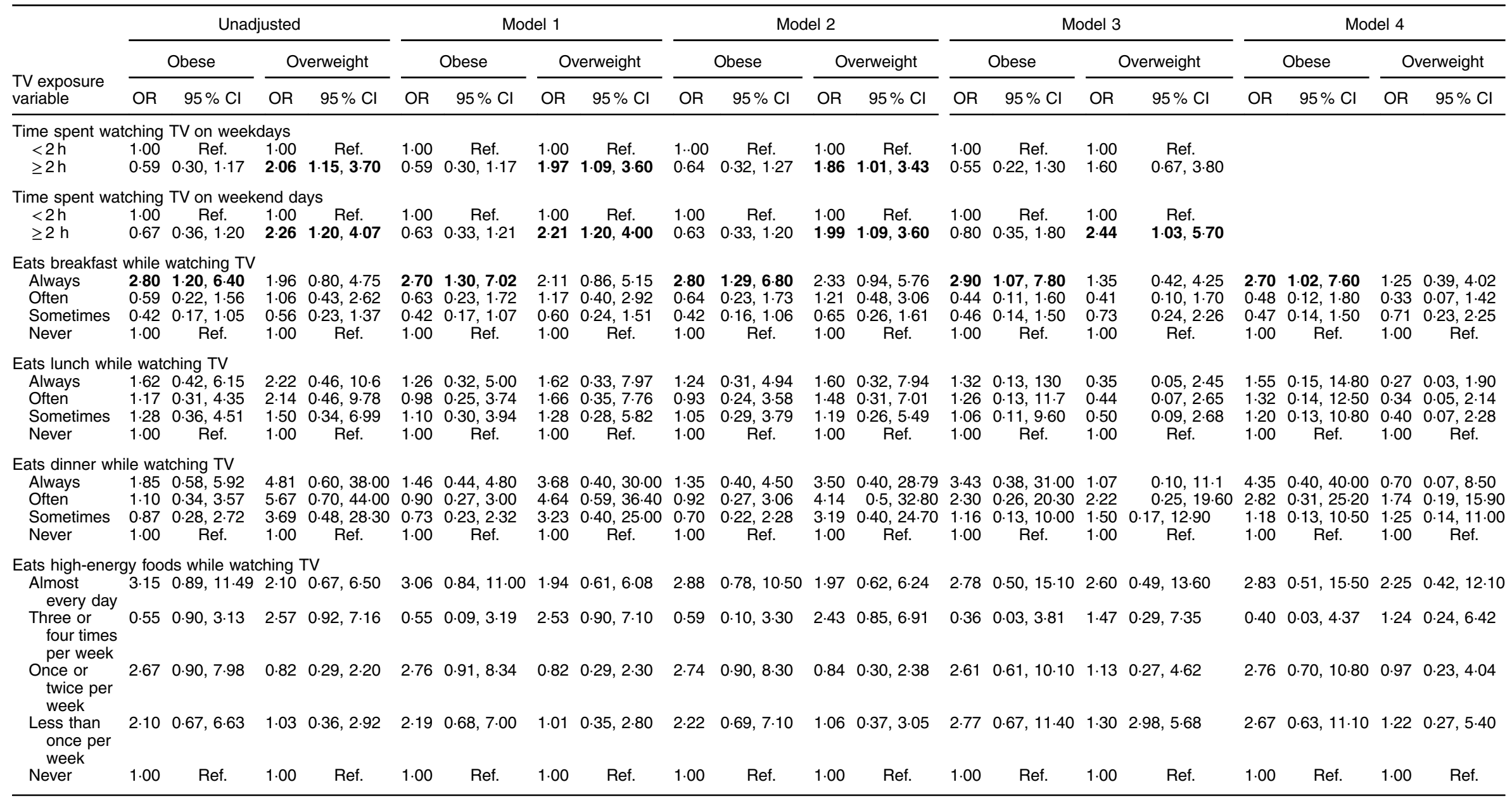

Ref., reference category.

birth order and income

Model 2 was adjusted for sex, birth order, income and physical activity.

Model 3 was adjusted for sex, birth order, income, physical activity and energy intake.

Model 4 was adjusted for sex, birth order, income, physical activity, energy intake and total TV viewing time.

Significant results $(P<0.05)$ are indicated in bold font 
compared with other mealtimes. Previous studies have shown that children who eat meals with their parents are encouraged to have healthier eating behaviours (i.e. better diet quality) $)^{(36)}$.

The type of TV programming may also influence diet quality and overall food intake. It is suggested that eating while watching TV can result in 'mindless eating'; that is, screen time can act as a distractor that can limit the capacity of an individual to monitor physiological signals associated with satiety, ultimately leading to an increase in energy intake ${ }^{(37)}$. Watching violent movies increases anxiety and preference for salty and fatty foods, while watching non-violent movies (e.g. romantic genre) results in a sleepy feeling and preference for sweet foods ${ }^{(38)}$. Previous studies have reported that children who watched happy movies were in a more 'mindless state' than children who watched neutral movies ${ }^{(39)}$. Although we did not distinguish the types of TV shows viewed while eating meals, Iranian TV channels show a wide range of children's programmes from 08.00 to 10.00 hours, and it is likely children ate their preferred sweeter carbohydratebased foods at breakfast while watching 'happy' children's shows. Future studies should observe TV programming during mealtimes and examine the underling biological mechanism for increased food intake during TV viewing.

The strengths of the present study include its large sample size and adjustment for a large number of confounding variables that have not been taken into account in previous studies. Limitations include: (i) the crosssectional design, which limits the ability to conclude a causal relationship between eating while watching TV and overweight/obesity; (ii) the lack of data regarding the types of programming that children watched while eating meals that may have influenced food intake and body composition; and (iii) only 410 parents marked which of the meals and snacks were eaten while watching TV. Future studies should consider other common screen-time modalities such as computer games and mobile phones.

\section{Conclusion}

In conclusion, our results show that repeated consumption of breakfast in front of the TV, independent of physical activity and total hours spent watching TV, is related to overweight/obesity among Iranian children. We suggest that limiting meals in front of the TV may reduce overweight/obesity among children and improve diet quality, but further clinical trials and/or longitudinal studies are needed to better understand the causal relationship between TV viewing behaviours and overweight/obesity.

\section{Acknowledgements}

Acknowledgements: This article has been extracted from S.G.'s master's thesis (code number 9888-84), funded by 
Shiraz University of Medical Sciences. The authors thank all those who helped with this study. Special thanks go to the participants and their parents for their kind cooperation. Financial support: This work was supported by Shiraz University of Medical Sciences, Shiraz, Iran (grant number 9888-84). The funder had no role in the design, analysis or writing of this article. Conflict of interest: The authors have no conflict of interest to declare. Authorship: S.G. contributed in all parts of the study. N.B. and J.O.T.d.Z. contributed to the study design, interpretation of data and revision. Z.H., R.B. and H.A. participated in collecting data and drafting the manuscript. M.S.-M. contributed to analysis and interpretation of data and revision. S.F. contributed in conception and design, analysis and interpretation of data, and critical revision. Ethics of buman subject participation: All procedures involving human participants were performed in accordance with the ethical standards of the institutional research ethics committee and with the 1964 Helsinki Declaration. The aim and study protocol were approved by the Research Ethics Committee of Shiraz University of Medical Sciences (number 94-01-84-9888). Informed consent was obtained from all parents or caregivers of children who participated in the study.

\section{References}

1. Ebbeling CB, Pawlak DB \& Ludwig DS (2002) Childhood obesity: public-health crisis, common sense cure. Lancet 360, 473-482.

2. Xie YJ, Stewart SM, Lam TH et al. (2014) Television viewing time in Hong Kong adult population: associations with body mass index and obesity. PLOS One 9, e85440.

3. Lawlor DA, Benfield L, Logue J, Tilling K et al. (2010) Association between general and central adiposity in childhood, and change in these, with cardiovascular risk factors in adolescence: prospective cohort study. BMJ 341, c6224.

4. Liang T, Kuhle S \& Veugelers PJ (2009) Nutrition and body weights of Canadian children watching television and eating while watching television. Public Health Nutr 12, 2457-2463.

5. Esmaili H, Bahreynian M, Qorbani M et al. (2015) Prevalence of general and abdominal obesity in a nationally representative sample of Iranian children and adolescents: the CASPIAN-IV Study. Iran J Pediatr 25, e401.

6. Smith L, Gardner B \& Hamer M (2015) Childhood correlates of adult TV viewing time: a 32-year follow-up of the 1970 British Cohort Study. J Epidemiol Community Health 69, 309-313.

7. Sisson SB, Shay CM, Broyles ST et al. (2012) Televisionviewing time and dietary quality among US children and adults. Am J Prev Med 43, 196-200.

8. Hu FB, Leitzmann MF, Stampfer MJ et al. (2001) Physical activity and television watching in relation to risk for type 2 diabetes mellitus in men. Arch Intern Med 161, 1542-1548.

9. Grontved A \& Hu FB (2011) Television viewing and risk of type 2 diabetes, cardiovascular disease, and all-cause mortality: a meta-analysis. JAMA 305, 2448-2455.

10. Vik FN, Bjørnarå HB, Øverby NC et al. (2013) Associations between eating meals, watching TV while eating meals and weight status among children, ages 10-12 years in eight European countries: the ENERGY cross-sectional study. Int J Behav Nutr Phys Act 10, 58.

11. Francis LA \& Birch LL (2006) Does eating during television viewing affect preschool children's intake? J Am Diet Assoc 106, 598-600.
12. Matheson DM, Killen JD, Wang Y et al. (2004) Children's food consumption during television viewing. Am J Clin Nutr 79, 1088-1094.

13. Wansink B \& van Kleef E (2014) Dinner rituals that correlate with child and adult BMI. Obesity (Silver Spring) 22, E91-E95.

14. Roos E, Pajunen T, Ray C et al. (2014) Does eating family meals and having the television on during dinner correlate with overweight? A sub-study of the PRO GREENS project, looking at children from nine European countries. Public Health Nutr 17, 2528-2536.

15. Motlagh ME, Kelishadi R, Amirkhani MA et al. (2011) Double burden of nutritional disorders in young Iranian children: findings of a nationwide screening survey. Public Health Nutr 14, 605-610.

16. Maalouf-Manasseh Z, Metallinos-Katsaras E \& Dewey KG (2011) Obesity in preschool children is more prevalent and identified at a younger age when WHO growth charts are used compared with CDC charts. J Nutr 141, 1154-1158.

17. Amiri P, Jalali-Farahani S, Zarkesh M et al. (2014) Reliability and validity of the Iranian version of the QAPACE in adolescents. Qual Life Res 23, 1797-1802.

18. Boyce W, Torsheim T, Currie C et al. (2006) The Family Affluence Scale as a measure of national wealth: validation of an adolescent self-report measure. Soc Indic Res 78, 473-487.

19. Van den Bulck J \& Van Mierlo J (2004) Energy intake associated with television viewing in adolescents, a cross sectional study. Appetite 43, 181-184.

20. Yilmaz G, Demirli Caylan N, Karacan C et al. (2015) An intervention to preschool children for reducing screen time: a randomized controlled trial. Child Care Health Dev 41, 443-449.

21. Kipping RR, Payne C \& Lawlor DA (2008) Randomised controlled trial adapting US school obesity prevention to England. Arch Dis Child 93, 469-473.

22. Fuller-Tyszkiewicz M, Skouteris H, Hardy LL et al. (2012) The associations between TV viewing, food intake, and BMI. A prospective analysis of data from the Longitudinal Study of Australian Children. Appetite 59, 945-948.

23. Hancox RJ, Milne BJ \& Poulton R (2004) Association between child and adolescent television viewing and adult health: a longitudinal birth cohort study. Lancet 364, 257-262.

24. Zhang G, Wu L, Zhou L et al. (2016) Television watching and risk of childhood obesity: a meta-analysis. Eur J Public Health 26, 13-18.

25. Peck T, Scharf RJ, Conaway MR et al. (2015) Viewing as little as 1 hour of TV daily is associated with higher change in BMI between kindergarten and first grade. Obesity (Silver Spring) 23, 1680-1686.

26. Bickham DS, Blood EA, Walls CE et al. (2013) Characteristics of screen media use associated with higher BMI in young adolescents. Pediatrics 131, 935-941.

27. Després JP (2016) Physical activity, sedentary behaviours, and cardiovascular health: when will cardiorespiratory fitness become a vital sign? Can J Cardiol 32, 505-513.

28. Hamilton MT, Hamilton DG \& Zderic TW (2004) Exercise physiology versus inactivity physiology: an essential concept for understanding lipoprotein lipase regulation. Exerc Sport Sci Rev 32, 161-166.

29. Henson J, Edwardson CL, Morgan B et al. (2014) Associations of sedentary time with fat distribution in a high-risk population. Med Sci Sports Exerc 47, 1727-1734.

30. Lissner L, Lanfer A, Gwozdz W et al. (2012) Television habits in relation to overweight, diet and taste preferences in European children: the IDEFICS study. Eur J Epidemiol 27, 705-715.

31. Dubois L, Farmer A, Girard M et al. (2008) Social factors and television use during meals and snacks is associated with higher BMI among pre-school children. Public Health Nutr 11, 1267-1279. 
32. Lipsky LM, Haynie DL, Liu DP et al. (2015) Trajectories of eating behaviors in a nationally representative cohort of US adolescents during the transition to young adulthood. Int J Behav Nutr Phys Act 12, 138.

33. Santiago-Torres M, Adams AK, Carrel AL et al. (2014) Home food availability, parental dietary intake, and familial eating habits influence the diet quality of urban Hispanic children. Child Obes 10, 408-415.

34. Hare-Bruun H, Nielsen BM, Kristensen PL et al. (2011) Television viewing, food preferences, and food habits among children: a prospective epidemiological study. BMC Public Health 11, 311.

35. Wildey MB, Pampalone SZ, Pelletier RL et al. (2000) Fat and sugar levels are high in snacks purchased from student stores in middle schools. J Am Diet Assoc 100, 319-322.
36. Poulos NS, Pasch KE, Springer AE et al. (2014) Is frequency of family meals associated with parental encouragement of healthy eating among ethnically diverse eighth graders? Public Health Nutr 17, 998-1003.

37. Fletcher E, Leech R, McNaughton S et al. (2015) Is the relationship between sedentary behaviour and cardiometabolic health in adolescents independent of dietary intake? A systematic review. Obes Rev 16, 795-805.

38. Mattar L, Zeeni N \& Bassil M (2015) Effect of movie violence on mood, stress, appetite perception and food preferences in a random population. Eur J Clin Nutr 69, 972-973.

39. Bevelander KE, Meiselman HL, Anschütz DJ et al. (2013) Television watching and the emotional impact on social modeling of food intake among children. Appetite $\mathbf{6 3}$, $70-76$. 